\title{
Vascular Endothelial Function in Patients with Coronary Slow Flow and the Effects of Nebivolol
}

\author{
Yilmaz Gunes¹, Hasan Ali Gumrukcuoglu², Serkan Akdag, Hakki Simsek², Musa Sahin², Mustafa Tuncer ${ }^{3}$ \\ Hisar Intercontinental Hospital, Cardiology Department ${ }^{1}$, Istanbul, Turkey; Yuzuncu Yil University, Faculty of Medicine, Cardiology Department ${ }^{2}$, \\ Van, Turkey; Medical Park Hospital, Cardiology Department ${ }^{3}$, Van, Turkey; Yuksek Ihtısas Research Hospital, Cardiology Department ${ }^{4}$, Van, Turkey
}

\begin{abstract}
Background: Brachial endothelial function has been associated with coronary slow flow (CSF). Increasing blood flow to brachial artery provokes endothelium to release nitric oxide (NO) with subsequent vasodilatation. Besides its $\beta 1$-blocker activity, nebivolol causes vasodilatation by increasing endothelial NO release.
\end{abstract}

Objective: To assess the effects of nebivolol on vascular endothelial function in patients with CSF.

Methods: Forty-six patients with CSF and 23 individuals with normal epicardial coronary arteries were examined with transthoracic echocardiography and brachial artery ultrasonography. The patients were reevaluated two months after treatment with aspirin or aspirin plus nebivolol.

Results: Patients with CSF had higher body mass index $(26.5 \pm 3.3$ vs. $23.8 \pm 2.8, p<0.001)$, mitral inflow isovolumetric relaxation time (IVRT) $(114.9 \pm 18.0 \mathrm{vs.} 95.0 \pm 22.0 \mathrm{msec}, \mathrm{p}<\mathbf{0 . 0 0 1})$ and lower left ventricular ejection fraction (LVEF) $(63.5 \pm 3.1 \%$ vs. $65.4 \pm 2.2, p=0.009)$, HDL-cholesterol $(39.4 \pm 8.5$ vs. $45.8 \pm 7.7 \mathrm{mg} / \mathrm{dL}, p=0.003)$ and brachial flow-mediated dilatation (FMD) $(6.1 \pm 3.9 \%$ vs. $17.6 \pm 4.5 \%, p<0.001)$. There were significant correlations between FMD and the presence of CSF $(r=0.800, p<0.001)$ and HDL-cholesterol $(r=0.349, p=0.003)$. Among Patients with CSF, although pretreatment mean FMD values were similar $(6.1 \pm 4.3 \%$ vs. $6.0 \pm, 6 \%, p=0.917)$ compared to aspirin alone group, posttreatment FMD was significantly higher in patients treated with aspirin plus nebivolol $(6.0 \pm 3.5 \%$ vs. $8.0 \pm 2.9 \%, p=0.047)$. Treatment with nebivolol was associated with a significant increase in FMD $(6.0 \pm 3.6$ to $8.0 \pm 2.9 \%, p=0.030$ ) whereas treatment with aspirin alone was not.

Conclusions: Endothelial function may be impaired in both coronary and brachial arteries in patients with CSF and nebivolol may be effective in the improvement of endothelial function in patients with CSF. (Arq Bras Cardiol $2011 ; 97(4): 275-280$ )

Keywords: Endothelium, vascular/physiology; regional blood flow; calcium channel blockers.

\section{Introduction}

Coronary slow flow (CSF) is a phenomenon characterized by delayed opacification of coronary arteries in the absence of epicardial occlusive disease, in which many etiological factors such as microvascular and endothelial dysfunction and small vessel disease have been implicated ${ }^{1-5}$.

The endothelium is of essential importance for the maintenance of vascular tone. It participates in the regulation of blood flow in response to changes in tissue and organ perfusion requirements. When blood flow increases through a vessel, the vessel dilates. This phenomenon has been called flow- mediated dilatation (FMD). Flow-mediated vasodilatation has been widely examined non-invasively by ultrasound-based measurements of brachial artery vasodilatory response to increasing blood flow reflecting shear stress

Mailing Address: Yilmaz Gunes •

Hisar Intercontinental Hospital, Cardiology Department - 9065-100 Istanbul, Turkey

E-mail: yilmazleman@yahoo.com

Manuscript received December 05, 2010, revised manuscript received March 04, 2011, accepted March 04, 2011. on the vascular wall. This stimulus makes the endothelium release nitric oxide (NO) with subsequent vasodilatation that can be imaged and quantitated as an index of vasomotor function ${ }^{6}$. Brachial endothelial function has also been found to be associated with $\mathrm{CSF}^{7,8}$. Nebivolol is a third-generation highly cardioselective $\beta 1$-adrenoceptor antagonist and it also affects the L-arginine/ NO pathway, causing vasodilatation by increasing endothelial NO release ${ }^{9}$.

This study aimed to investigate the effects of nebivolol treatment on FMD in patients with CSF.

\section{Methods}

Forty-six patients with angiographically proven CSF but normal epicardial coronary arteries and 23 healthy individuals were selected from patients who had undergone diagnostic coronary arteriography because of suspected coronary artery disease and were found to have normal epicardial coronary arteries without CSF. Coronary slow flow was defined according to the TIMI frame count (TFC) method, and the individuals with a TFC greater than 2 standard deviations (SD) 
from the published normal range for the particular vessel were accepted as having $\mathrm{CSF}^{10}$. Patients with a history of congestive heart failure, coronary artery disease including spasm, plaque, or ectasia, valvular heart disease, hyperthyroidism, chronic obstructive pulmonary disease, ventricular preexcitation, atrioventricular conduction abnormalities and those taking medications known to alter cardiac conduction were excluded from the study. The patients underwent echocardiography and brachial artery ultrasound evaluation before and two months after treatment with aspirin alone or aspirin plus nebivolol. The study was approved by the Yuzuncu Yil University Medical Faculty ethic committee according to the Declaration of Helsinki and the patients gave written informed consent.

The echocardiography examination was performed at rest, with the patient at left lateral decubitis position, using a commercially available echocardiography device (Vivid 3, General Electric) with a $3 \mathrm{MHz}$ transducer, by two experienced echocardiographers who were blinded to the clinical data. Using M-mode echocardiography, long-axis measurements were obtained distally to the mitral valve leaflets according to current recommendations ${ }^{11}$. Left ventricular ejection fraction was calculated via modified biplane Simpson's method from apical four and two chamber views. The pulsed Doppler sampling volume was placed between the tips of the mitral valve leaflets to obtain maximum filling velocities. Early diastolic flow (E), atrial contraction signal $(\mathrm{A})$ and $\mathrm{E}$ deceleration time (DT) were measured. Isovolumetric relaxation time (IVRT) was determined as the interval between the end of the aortic outflow and the start of the mitral inflow signal.

Changes in right brachial artery diameter in response to reactive hyperemia were measured using high-resolution ultrasound system (Vivid 3, General Electric) with a $7-\mathrm{MHz}$ linear-array transducer as previously described ${ }^{12}$. After baseline images of the brachial artery were obtained, a pneumatic tourniquet placed around the forearm distally to the target artery was inflated to a pressure of $250 \mathrm{~mm} \mathrm{Hg}$, and the pressure was held for 5 minutes. Increased flow was induced by sudden cuff deflation. A second scan was performed continuously for 30 seconds before and for 90 seconds after cuff deflation. Fifteen minutes later a further resting scan was recorded to confirm the vessel recovery. Diameter of the brachial artery was measured from the anterior to posterior interface between the media and adventitia at the end of diastole, incident with the $\mathrm{R}$ wave on a continuously recorded ECG. Diameters for 4 cardiac cycles were determined from images, and the measurements were averaged. The scans were evaluated by a single observer blinded to the clinical data of the patients. FMD was calculated as percentage increase in arterial diameter during reactive hyperemia and was used as an index of endothelium-dependent vasodilatation.

\section{Statistics}

Data were presented as mean \pm standard deviation (SD). Using an SPSS package 10.0 (SPSS Inc., Chicago, Illinois, USA) differences in mean values between groups were assessed using Student t- test and with Mann-Whitney's U-test for variables without normal distribution. Categorical variables were compared by chi square test. The changes in parameters after treatment were compared using paired t-test.
Pearson correlation analysis was used for the assessment of relation between variables. A two-tailed $p$ value $<0.05$ was considered significant.

\section{Results}

Coronary slow flow was observed in 3 vessels in 11 (23.9\%) patients, in 2 vessels in 21 (45.7\%) patients and in one vessel in 14 (30.4\%) patients. Left anterior descending artery (LAD) was affected in 37 (80.4\%) patients, circumflex artery (CX) in 25 (54.3) patients and right coronary artery (RCA) in 28 (60.9\%) patients. Thirty-two (69.6\%) patients had angina symptoms and $14(30.4 \%)$ had positive treadmill exercise test. Patients with CSF had higher body mass index, IVRT and lower HDLcholesterol and FMD. Although it was within normal limits, LVEF was slightly but significantly lower among CSF patients (Table 1). There were significant correlations between FMD and the presence of CSF $(r=0.800, p<0.001)$, BMI $(r=-0.381$, $p=0.001), \operatorname{HDL}(r=0.349, p=0.003)$. Although baseline mean FMD values were similar $(6.1 \pm 4.3 \%$ vs. $6.0 \pm 3.6 \%$, $\mathrm{p}=0.917$ ), compared to aspirin alone group, posttreatment FMD was significantly higher in patients treated with aspirin plus nebivolol $(6.0 \pm 3.5 \%$ vs. $8.0 \pm 2.9, p=0.047)$. (Table 2, Figure 1). Treatment with nebivolol was associated with significant decrease in heart rate and significant increase in FMD whereas treatment with aspirin alone was not (Table 2). All the patients were free of angina after treatment.

\section{Discussion}

We have found that CSF is associated with impaired brachial artery vascular endothelial function and LV functions. Compared with aspirin alone, addition of nebivolol to treatment improved brachial endothelial function.

According to previous studies, we found that vascular endothelial functions were impaired in patients with $\mathrm{CSF}^{7,8,13}$. Endothelial dysfunction appears to be a generalized process and can be detected in coronary and peripheral arteries before the development of atherosclerosis ${ }^{14}$. Brachial artery imaging with high-resolution ultrasound during reactive hyperemia is an accepted method of assessing endothelium-dependent vasomotion, and abnormalities in peripheral vasomotor function have been correlated with the presence of coronary arterial endothelial dysfunction ${ }^{15}$. Moreover, in patients with microvascular angina, an impaired forearm vasodilator response to ischemia has been observed, suggesting that the vascular involvement is not restricted to the coronary circulation but is part of a generalized vasomotor disturbance ${ }^{16}$.

Coronary slow flow has been reported to be associated with low LV systolic and diastolic function ${ }^{17-19}$ and low HDL and high triglyceride levels ${ }^{13}$. We have also found that compared to control group, IVRT (a diastolic function parameter) was longer and HDL levels were lower in patients CSF and they were significantly correlated with FMD. Although it was within normal limits, LVEF was slightly but significantly lower among CSF patients in this study. However, Barutcu et al ${ }^{20}$ found no differences in left ventricular systolic function between patients with CSF and the control group. In another study, Sezgin et $\mathrm{al}^{17}$ found diastolic filling abnormalities but normal LV systolic 


\section{Original Article}

function in patients with CSF. We have also found that patients with CSF had impaired diastolic and regional LV functions ${ }^{21}$.

Diastolic dysfunction without systolic dysfunction may appear at an early stage of myocardial ischemia in patients with coronary artery disease, compatible with the fact that left ventricular diastolic functions are more susceptible to ischemia than systolic functions ${ }^{22}$. Several studies have demonstrated that CSF is associated with myocardial ischemia, but data are limited on how the left ventricular functions are affected by this disease $\mathrm{e}^{2,3,5,23}$. The association between low HDL and endothelial dysfunction has been documented ${ }^{24}$ and in patients with CSF reduced brachial, FMD was found to be associated with low HDL levels ${ }^{13}$.

Previous studies have suggested abnormally high small vessel resistance and increased microvascular tone as the cause of $\mathrm{CSF}^{1,4,25}$. Sympathetic stimulation has a major role in regulating coronary arterial tonus. Patients with CSF had higher adrenalin and noradrenalin levels compared to patients with normal coronary flow and TIMI frame count was reported to be positively correlated with adrenalin and noradrenalin levels suggesting that adrenergic hyperactivity might have an impact on CSF pathogenesis ${ }^{26}$. It is noteworthy that only one third of the patients with CSF fulfilled the criteria on a repeat angiographic study ${ }^{27}$. Although the exact mechanism for this dynamic response is not well elucidated, it is postulated that this dynamic increase in microvascular tone may be due to microvascular spasm resulting in recurrence of symptoms. Therefore, drugs acting on the sympathetic and neuroendocrine system having coronary vasodilatory effects may be useful in the treatment of this entity. Previous clinical studies using coronary flow measurements suggest that non-selective beta-adrenergic antagonists are associated with an increase in coronary vascular resistance during hyperemia and, therefore, reduce coronary flow reserve ${ }^{28,29}$. This phenomenon has been attributed to the unopposed alpha-adrenergic vasomotor tone. However, selective betablockers like metoprolol have been confirmed to improve myocardial perfusion by increasing coronary flow reserve ${ }^{30}$.

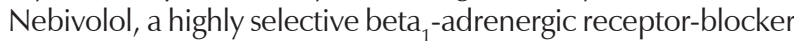
with NO-modulating properties, may be especially useful for improving coronary flow reserve due to its vasodilating properties on the small and large coronary arteries ${ }^{30}$. The marked vasodilating effect of nebivolol in human coronary microvessels is well established under in vitro conditions ${ }^{31,32}$. In addition, intracoronary nebivolol has been associated with a significant increase in coronary flow reserve both in patients with coronary artery disease and patients with normal coronary arteries33. Furthermore, it has been shown that nebivolol may be effective for improvement of LV functions and symptoms and brachial FMD in patients with $\mathrm{CSF}^{20,34,35}$. Improvement of FMD with nebivolol in this study may be associated with the fact that endothelial dysfunction may be a generalized process and can be detected both in coronary and peripheral arteries.

As far as we know, there is no study investigating the effects of aspirin in CSF. In this study, we did not find any improvement in FMD with aspirin. However, both patients

Table 1 - Comparison of the baseline clinical characteristics of the study population.

\begin{tabular}{lccc}
\hline & Patients with NCA $(\mathbf{n}=\mathbf{2 3})$ & Patients with CSF $(\mathbf{n}=46)$ & p value \\
\hline Age, years & $48,5 \pm 9,4$ & $52,1 \pm 10,6$ & 0,179 \\
\hline Hypertension & $9(19,6 \%)$ & $6(26,1 \%)$ & 0,550 \\
\hline Diabetes mellitus & 0 & $3(6,5 \%)$ & 0,546 \\
\hline Smoking & $10(43,5 \%)$ & $17(37,0 \%)$ & 0,798 \\
\hline BMl, $\mathrm{kg} / \mathrm{m}^{2}$ & $23,8 \pm 2,8$ & $26,5 \pm 3,3$ & 0,001 \\
\hline Total cholesterol, $\mathrm{mg} / \mathrm{dL}$ & $188,5 \pm 26,2$ & $185,5 \pm 39,4$ & 0,748 \\
\hline LDL-cholesterol, $\mathrm{mg} / \mathrm{dL}$ & $107,9 \pm 13,9$ & $115,8 \pm 24,0$ & 0,149 \\
\hline HDL-cholesterol, $\mathrm{mg} / \mathrm{dl}$ & $45,8 \pm 7,7$ & $39,4 \pm 8,5$ & 0,003 \\
\hline Triglyceride, $\mathrm{mg} / \mathrm{dl}$ & $161,3 \pm 51,2$ & $178,8 \pm 110,5$ & 0,750 \\
\hline LAD-TFC & $26,7 \pm 6,4$ & $47,2 \pm 19,8$ & $<0,001$ \\
\hline CX-TFC & $25,7 \pm 2,4$ & $52,0 \pm 19,9$ & $<0,001$ \\
\hline RCA-TFC & $23,8 \pm 2,6$ & $<3,9 \pm 25,8$ & $<0,001$ \\
\hline LVEF, $\%$ & $65,4 \pm 2,2$ & $63,5 \pm 3,1$ & 0,009 \\
\hline DT, msec & $210,6 \pm 53,9$ & $216,8 \pm 29,4$ & 0,541 \\
\hline IVRT, msec & $95,0 \pm 22,0$ & $114,9 \pm 18,0$ & $<0,001$ \\
\hline Heart rate, bpm & $77,1 \pm 8,2$ & $77,5 \pm 8,2$ & 0,836 \\
\hline FMD, $\%$ & $17,6 \pm 4,5$ & $6,1 \pm 3,9$ & $<0,001$ \\
\hline
\end{tabular}

NCA - Normal coronary arteries; CSF - Coronary slow flow; LAD - Left anterior descending artery; TFC - TIMI frame count; Cx - Circumflex artery; RCA - Right coronary artery artery; LVEF - Left ventricular ejection fraction; DT - Decelaration time; IVRT - Isovolumetric relaxation time; FMD - flow-mediated dilatation. 
treated with either aspirin alone or aspirin plus nebivolol were free of angina after treatment. This finding may be related to the dynamic nature of CSF. Recurrent events may be secondary to dynamic increase of microvascular spasm secondary to microvascular spasm ${ }^{2}$. Only one third of the patients with CSF were detected to have CSF on a repeat angiographic study ${ }^{36}$.

Table 2 - Comparison of pre- and posttreatment values of some variables in patients with CSF

\begin{tabular}{|c|c|c|c|c|}
\hline & & Aspirin & Aspirin plus nebivolol & $p$ value \\
\hline \multirow{2}{*}{ LVEF, \% } & Pretreatment & $63,6 \pm 2,8$ & $63,3 \pm 3,5$ & 0,713 \\
\hline & Posttreatment & $63,8 \pm 2,7$ & $63,4 \pm 3,5$ & 0,672 \\
\hline \multirow{2}{*}{ DT, msec } & Pretreatment & $222,6 \pm 31,5$ & $211,0 \pm 26,6$ & 0,184 \\
\hline & Posttreatment & $220,5 \pm 27,1$ & $212,4 \pm 28,5$ & 0,327 \\
\hline \multirow{2}{*}{ IVRT, msec } & Pretreatment & $118,1 \pm 19,7$ & $111,6 \pm 15,9$ & 0,227 \\
\hline & Posttreatment & $115,3 \pm 16,3$ & $108,8 \pm 14,9$ & 0,166 \\
\hline \multirow{2}{*}{ Heart rate, bpm } & Pretreatment & $77,5 \pm 6,2$ & $77,5 \pm 9,9$ & 0,972 \\
\hline & Posttreatment & $76,3 \pm 8,7$ & $70.5 \pm 6,2 \mathrm{a}$ & 0,012 \\
\hline \multirow{2}{*}{ FMD, $\%$} & Pretreatment & $6,1 \pm 4,3$ & $6,0 \pm 3,6$ & 0,917 \\
\hline & Posttreatment & $6,0 \pm 3,5$ & $8.0 \pm 2.9 b$ & 0,047 \\
\hline
\end{tabular}

LVEF - Left ventricular ejection fraction; DT - Decelaration time; IVRT - Isovolumetric relaxation time; FMD - flow-mediated dilatation; ${ }^{a} p<0.001$ post- vs. pretreatment, ${ }^{c} p=0.030$ post- $v$ s. pretreatment.

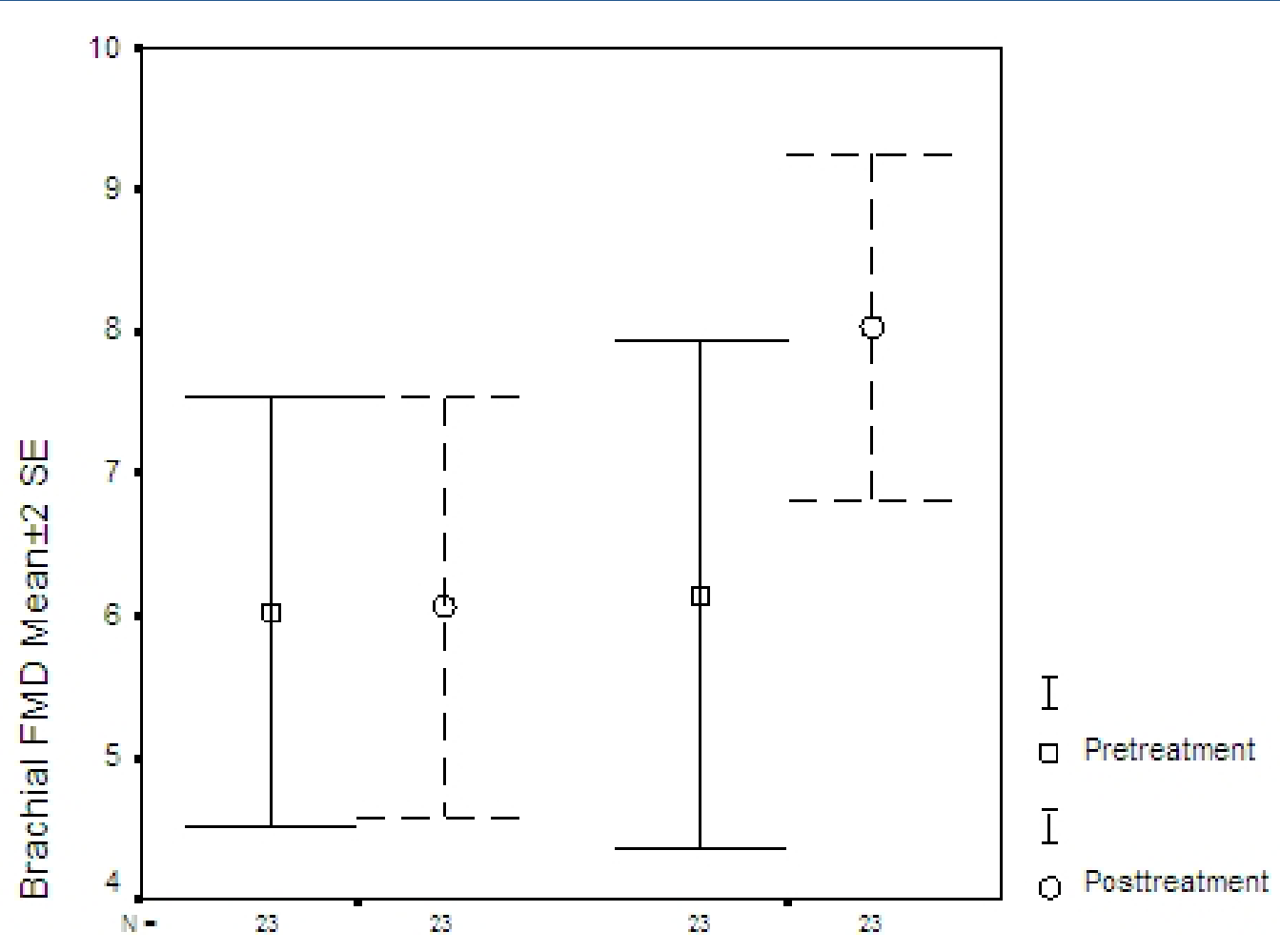

Aspirin Nebivolol+aspirin

Figure 1 -Error bar for FMD; baseline and two months after treatment with aspirin or aspirin plus nebivolol. SE: Standard error, FMD: flow-mediated dilatation. 


\section{Original Article}

\section{Limitations}

The small number of patients included in this study is the main limitation. Larger and longer-term follow-up should strengthen the value of the results. Control angiography to assess the effects of nebivolol on TFC was not performed due to ethical concerns.

\section{Conclusion}

Endothelial function may be impaired in both coronary and brachial arteries in patients with CSF and nebivolol may be effective in the improvement of brachial FMD, an index of endothelial function, in patients with CSF.

\section{Referencias}

1. Tambe AA, Demany MA, Zimmerman HA, Mascarenhas E. Angina pectoris and slow flow velocity of dye in coronary arteries - a new angiographic finding. Am Heart J. 1972;84(1):66-71.

2. Singh S, Kothari SS, Bahl VK. Coronary slow flow phenomenon: an angiographic curiosity. Indian Heart J. 2004;56(6):613-7.

3. Pekdemir H, Cin VG, Cicek D, Camsari A, Akkus N, Döven O, et al. Slow coronary flow may be a sign of diffuse atherosclerosis. Contribution of FFR and IVUS. Acta Cardiol. 2004;59(2):127-33

4. Mangieri E, Macchiarelli G, Ciavolella M, Barillà F, Avella A, Martinotti A, et al. Slow coronary flow: clinical and histopathological features in patients with otherwise normal epicardial coronary arteries. Cathet Cardiovasc Diagn. 1996;37(4):375-81.

5. Beltrame JF, Limaye SB, Horowitz JD. The coronary slow flow phenomenon - a new coronary microvascular disorder. Cardiology. 2002;97(4):197-202.

6. Agewall S. Is impaired flow-mediated dilatation of the brachial artery a cardiovascular risk factor? Curr Vasc Pharmacol. 2003;1(2):107-9.

7. Teragawa H, Ueda K, Matsuda K, Kimura M, Higashi Y, Oshima T, et al. Relationship between endothelial function in the coronary and brachial arteries. Clin Cardiol. 2005;28(10):460-6.

8. Sezgin AT, Sigirci A, Barutcu I, Topal E, Sezgin N, Ozdemir R, et al. Vascular endothelial function in patients with slow coronary flow. Coron Artery Dis. 2003;14(2):155-61.

9. Ritter JM. Nebivolol: endothelium-mediated vasodilating effect. J Cardiovasc Pharmacol. 2001;38(Suppl. 3):S13-6.

10. Gibson CM, Cannon CP, Delay WL, Dodge JT Jr, Alexander B Jr, Marble SJ, et al. TIMI frame count: a quantitative method of assessing coronary artery flow. Circulation. 1996;93(5):879-83.

11. Lang RM, Bierig M, Devereux RB, Flachskampf FA, Foster E, Pellikka PA, et al; Chamber Quantification Writing Group; American Society of Echocardiography's Guidelines and Standards Committee; European Association of Echocardiography. Recommendations for chamber quantification: a report from the American Society of Echocardiography's Guidelines and Standards Committee and the Chamber Quantification Writing Group, developed in conjunction with the European Association of Echocardiography, a branch of the European Society of Cardiology. J Am Soc Echocardiogr. 2005;18(12):1440-63.

12. Stroes ESG, Koomans HA, de Bruin TWA, Rabelink TJ. Vascular function in the forearm of hypercholesterolemic patients off and on lipid-lowering medication. Lancet. 1995;346(8973):467-71.

13. Sezgin AT, Barutcu I, Sezgin N, Gullu H, Esen AM, Acikgoz N, et al. Contribution of plasma lipid disturbances to vascular endothelial function in patients with slow coronary flow. Angiology. 2007;57(6):694-701.

\section{Potential Conflict of Interest}

No potential conflict of interest relevant to this article was reported.

\section{Sources of Funding}

There were no external funding sources for this study.

\section{Study Association}

This study is not associated with any post-graduation program.
14. Heitzer T, Schlinzing T, Krohn K, Meinertz T, Münzel T. Endothelial dysfunction, oxidative stress and risk of cardiovascular events in patients with coronary artery disease. Circulation. 2001;104(22):2638-40.

15. Anderson TJ, Uehata A, Gerhard MD, Meredith IT, Knab S, Delagrange $D$, et al. Close relation of endothelial function in the human coronary and peripheral circulations. J Am Coll Cardiol. 1995;26(5):1235-41.

16. Sax FL, Cannon RO, Hanson C, Epstein SE. Impaired forearm vasodilator reserve in patients with microvascular angina. Evidence of generalized disorder of vascular function? N Engl J Med. 1987;317(22):1366-70.

17. Sezgin AT, Topal E, Barutcu I, Ozdemir R, Gullu H, Bariskaner E, et al. Impaired left ventricle filling in slow coronary flow phenomenon: an echoDoppler Study. Angiology. 2005;56(4):397-401.

18. Cannon RO 3rd, Bonow RO, Bacharach SL, Green MV, Rosing DR, Leon MB, et al. Left ventricular dysfunction in patients with angina pectoris, normal epicardial coronary arteries, and abnormal vasodilator reserve. Circulation. $1985 ; 71(2): 218-26$.

19. Sevimli S, Buyukkaya E, Gundogdu F, Arslan S, Aksakal E, Gürlertop Y, et al. Left ventricular function in patients with coronary slow flow: a tissue Doppler study. Arch Turkh Soc Cardiol. 2007;35:360-5.

20. Gunes Y, Tuncer M, Guntekin U, Ceylan Y, Sahin M, Simsek H. Regional functions of the left ventricle in patients with coronary slow flow and the effects of nebivolol. Ther Adv Cardiovasc Dis. 2009;3(6):441-6.

21. Barutcu I, Sezgin AT, Sezgin N, Gullu H, Esen AM, Topal E, et al. Elevated plasma homocysteine level in slow coronary flow. Int J Cardiol. $2005 ; 101(1): 143-5$.

22. Labovitz AJ, Lewen MK, Kern M, Vandormael M, Deligonul U, Kennedy $\mathrm{H}$. Evaluation of left ventricular systolic and diastolic dysfunction during transient myocardial ischemia produced by angioplasty. J Am Coll Cardiol. 1987;10(4):748-55.

23. Cesar LA, Ramires JA, Serrano Junior CV, Meneghetti JC, Antonelli RH, daLuz PL, et al. Slow coronary run-off in patients with angina pectoris: clinical significance and thallium-201 scintigraphic study. Braz J Med Biol Res. 1996;29(5):605-13.

24. Kuhn FE, Mohler ER, Satler LF, Reagan K, Lu DY, Rackley CE. Effects of highdensity lipoprotein on acetylcholine-induced coronary vasoreactivity. Am J Cardiol. 1991;68(15):1425-30.

25. Mosseri M, Yarom R, Gotsman MS, Hasin Y. Histologic evidence for smallvessel coronary artery disease in patients with angina pectoris and patent large coronary arteries. Circulation. 1986;74(5):964-72.

26. Yazici M, Demircan S, Durna K, Sahin M. The role of adrenergic activity in slow flow coronary flow and its relationship to TIMI frame count. Angiology. 2007;58(4):393-400. 
27. Beltrame JF, Limaye SB, Wuttke RD, Horowitz JD. Coronary hemodynamic and metabolic studies of the coronary slow flow phenomenon. Am Heart J. 2003;146(1):84-90.

28. Robertson RM, Wood AJ, Vaughn WK, Robertson D. Exacerbation of vasotonic angina pectoris by propranolol. Circulation. 1982;65(2):281-5.

29. Kern MJ, Ganz P, Horowitz JD, Gaspar J, Barry WH, Lorell BH, et al. Potentiation of coronary vasoconstriction by beta-adrenergic blockade in patients with coronary artery disease. Circulation. 1983;67(6):1178-85.

30. Billinger M, Seiler C, Fleisch M, Eberli FR, Meier B, Hess OM. Do betaadrenergic blocking agents increase coronary flow reserve? J Am Coll Cardiol. 2001;38(7):1866-71.

31. Dessy C, Moniotte S, Ghisdal P, Havaux X, Noirhomme P, Balligand JL. Endothelial beta3-adrenoceptors mediate vasorelaxation of human coronary microarteries through nitric oxide and endothelium-dependent hyperpolarization. Circulation. 2004;110(8):948-54.
32. Dessy C, Saliez J, Ghisdal P, Daneau G, Lobysheva II, Frérart F, et al Endothelial ß-3-adrenoreceptors mediate nitric oxide-dependent vasorelaxation of coronary microvessels in response to the third-generation ß -blocker nebivolol. Circulation. 2005;112(8):1198-205.

33. Togni M, Vigorito F, Windecker S, Abrecht L, Wenaweser P, Cook S, et al. Does the b-blocker nebivolol increase coronary flow reserve? Cardiovasc Drugs Ther. 2007;21(2):99-108.

34. Gunes Y, Tuncer M, Guntekin U, Ceylan Y. The effects of nebivolol on P wave duration and dispersion in patients with coronary slow flow. Anadolu Kardiyol Derg. 2009;9(4):290-5

35. Albayrak S, Ordu S, Yuksel H, Ozhan H, Yazgan O, Yazıcı M. Efficacy of nebivolol on flow mediated dilatation in patients with slow coronary flow. Int Heart J. 2009;50(5):545-53.

36. Beltrame JF, Limaye SB, Wuttke RD, Horowitz JD. Coronary hemodynamic and metabolic studies of the coronary slow flow phenomenon. Am Heart J. $2003 ; 146(1): 84-90$ 